\title{
ROBUST CLUSTERING OF ACOUSTIC EMISSION SIGNALS USING THE KOHONEN NETWORK
}

\author{
Vahid Emamian, Mostafa Kaveh, Ahmed H. Tewfik \\ Department of Electrical and Computer Engineering \\ University of Minnesota, Minneapolis, MN 55455, USA
}

\begin{abstract}
Acoustic emission-based techniques are promising for nondestructive inspection of mechanical systems. For reliable automatic fault monitoring, it is important to identify the transient crack-related signals in the presence of strong timevarying noise and other interference. In this paper we propose the application of the Kohonen network for this purpose. The principal components of the short-time Fourier transforms of the data were applied to the input of the network. The clustering results confirm the capability of the Kohonen network for reliable source identification of acoustic emission signals, assuming enough care has been taken in implementing the training algorithm of the network.
\end{abstract}

\section{INTRODUCTION}

Acoustic emission (AE) is an ultrasonic wave emitted from the deformation of materials. Specifically, AE is the transient wave resulting from the sudden release of stored energy during a deformation and failure process, such as fretting or crack growth in a material [2]. By using a surface-mounted transducer, which is sensitive to displacement or velocity, the released energy can be detected. An AE signal, recorded in a material test, conveys useful information about the fatigue behavior of a specimen, and is one of the several promising nondestructive inspection methods for automatic fault monitoring in mechanical systems. Usage of AE-based fault monitoring techniques can avoid costly and dangerous consequences in industrial and aeronautical applications, and increase the reliability and safety of the processes. In any AE-based monitoring, the recorded signal, not only contains damage-related sources such as fracture, slip, corrosion and crack, but also very strong interfering sources, e.g. electromagnetic interference, fretting, vibration noise, etc. These spurious sources make the automatic diagnosis a difficult task, and hence efforts should be made to develop robust source identification techniques.

As an alternative approach to standard pattern recognition and signal processing techniques, neural networks offer high capability in signal classification, based on automated learning procedures for massively parallel networks of simple processing elements [3] [11]. Neural network classifiers produce highly nonlinear decision surfaces, discovering structures and patterns among data, and have the generalization capability of correct classification of unobserved data. These features make neural networks attractive tools for identifying damage-related $\mathrm{AE}$ sources in a highly noisy environment.
Over the past few years, extensive research has been conducted on AE signal detection, classification, and localization at the University of Minnesota [6] [5] [4] [1] [12]. In this paper, we propose the application of the Kohonen network for clustering the crack-related AE signals. Since the data is not presented with a set of input-output pairs, we cannot use a supervised method. Hence we use the Kohonen network, because it is an unsuper-vised method in which the network is presented with only the input samples and samples are grouped into classes which are self-similar [8].

\section{DESCRIPTION OF COLLECTED DATA}

In this paper we have used two different sets of data: in-flight and multi-sensor laboratory data.

\subsection{In-flight data}

The rotor acoustic monitoring system (RAMS) was developed by Honeywell. More than 16 hours of flight data were recorded from eight piezoelectric sensors mounted on one of the rotor arms. A piezoelectric pinger is mounted on the connection link of the rotor arm to simulate micro-crack generation. When the pinger is ON, a small pressure proportional to the control voltage is added to the connection link. When the pressure is released, a transient acoustic signal is emitted from this area. Fig. 1 shows a segment of the time domain signal. This set of data was recorded in eight vectors, where each vector corresponds to a sensor. The sampling rate was $2 \mathrm{MHz}$.

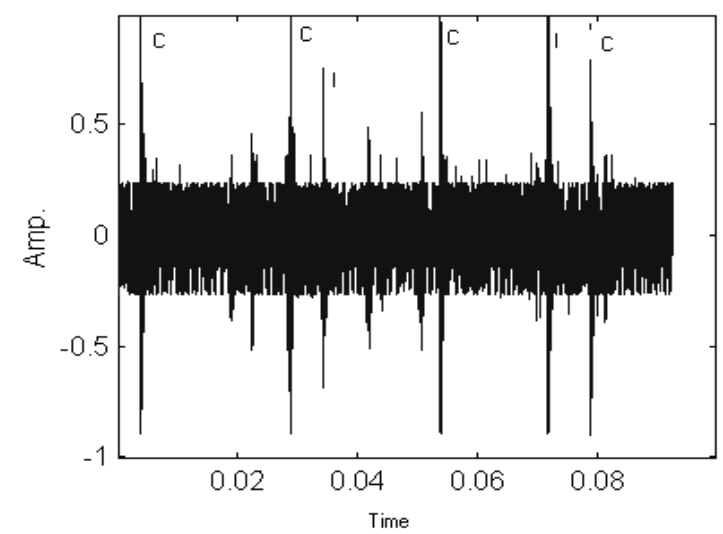

Figure 1. A segment of time domain signal from sensor 2 C: Crack I: Interference 


\subsection{Multi-sensor laboratory data}

A fatigue test was conducted at the Georgia Institute of Technology on a specimen to acquire multi-sensor data under a laboratory-controlled environment. The experimental setup is shown in Fig. 2. During the fatigue experiment, the specimen is stretched to generate the micro-crack in the central notch. Four sensors were arranged in specific positions such that transient acoustic signals emitted from the notch could propagate to the sensors at nearly the same time. The particular arrangement of the sensors is one of the most important criteria to distinguish the crack-related AE signal from other transient interference as well as multiple reflections of the original AE signal.

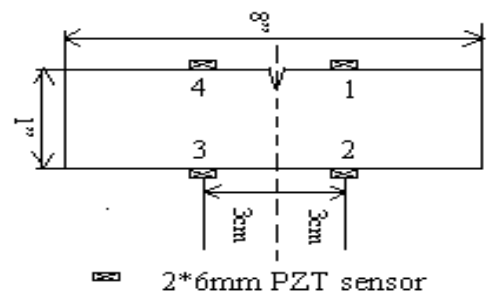

Figure 2. Experimental setup for generating the multi-sensor laboratory data at Georgia Tech.

During the AE dormant period, the micro-activities are accumulating or building up to overcome the threshold of the next higher energy level fracture. Spurious noise due to electrical-mechanical interference (EMI) can be largely eliminated with a proper system configuration, i.e., filter and threshold setting. AE events are generated from micro-fractures, which emit waves that are of similar amplitude to the noise level in the system. The micro-activities during this initial stage are basically the uniform growth and distribution of small sub-grainsized cracks. The total number of events for each sensor is 941 . These events were divided into four stages according to the event count history shown in Fig. 3. The first stage includes 508 events, the second stage has 108 events, the third stage contains 218 events, and the fourth stage has 107 events. Stage 1 correspondes to the initial micro-crack generation, and in stage 4 the cracks become visible. This set of data has been stored in four matrices, where each matrix corresponds to a sensor. Each column of a matrix contains 2048 samples of an AE event. The sampling rate was $25 \mathrm{MHz}$.

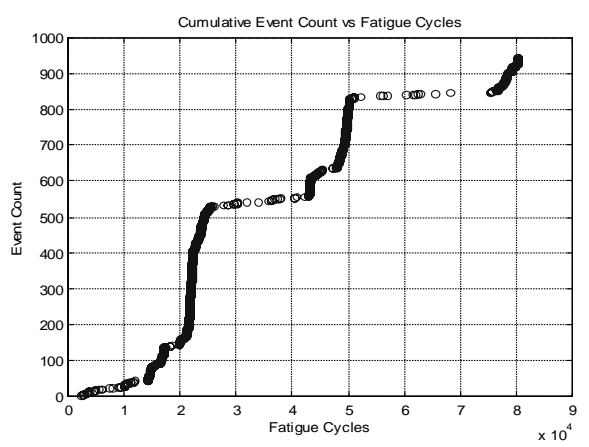

Figure 3. Event counts versus fatigue cycles.

\section{DATA PREPROCESSING AND FEATURE EXTRACTION-REDUCTION}

Since the main AE-related spectral information is concentrated in the $20 \mathrm{kHz}-1 \mathrm{MHz}$ frequency range, first we used a bandpass filter to remove undesired spectral data from the signals. For the multi-sensor laboratory data, we also did a downsampling by a factor of eight to reduce the amount of oversampled data.

Frequency-domain information is promising for source identification of AE signals. Therefore, we computed the 1024point and 256-point short time Fourier transform (STFT) of the in-flight and laboratory data, respectively. We discarded the second half of all the STFT vectors because of the symmetry property of the Fourier transform of real signals. Due to the large size of the feature vectors (512 and 128 for the first and the second data set, respectively), we carried out a principal component analysis (PCA) to reduce the dimensionality of data without any significant lost of information [13] [3]. Consequently, the size of all the feature vectors for both data sets was reduced to five, because the first five principal components contain more than $90 \%$ of the energy. Finally, the five-dimensional output of the PCA block was applied to the self-organizing map (SOM) Kohonen network for clustering the AE signals. Fig. 4 shows the block diagram of the above procedures.

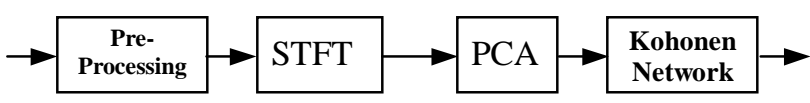

Figure 4. The block diagram of preprocessing, feature extraction reduction, and clustering procedures.

\section{IMPLEMENTATION OF THE KOHONEN NETWORK}

Consider a $k$-input, $M$-by- $N$ Kohonen network, where each neuron has a $k$-dimensional weight vector $W_{i}, i=1,2, \ldots, M N$. The training steps for the network are as follows [8]:

1. Initialize the network by assigning small random values $\left\{m_{1}, m_{2}, \ldots, m_{k}\right\}$ to the weight vector of each neuron:

$$
W_{i}(0)=\left[m_{1} m_{2} \cdots m_{k}\right], \quad i=1,2, \cdots, M N,
$$

where $W_{i}(0)$ refers to the initial value of $W_{i}$. Let $n=0$, where $n$ is the training step.

2. From the set of $k$-dimensional training vectors, choose a vector as the initial input vector $X(n)=\left[\begin{array}{llll}x_{1} & x_{2} & \cdots & x_{k}\end{array}\right]$. Then calculate the Euclidean distance between the weight of all neurons and $X(n)$ :

$$
D_{i}(n)=\left\|X(n)-W_{i}(n)\right\|, \quad i=1,2, \cdots, M N .
$$

3. Select the $j^{*}$-th neuron as the winner neuron if $D_{j}$ is minimum among $D_{j}$ 's, i.e.:

$$
j^{*}=\arg \min D_{j}, \quad j=1,2, \cdots, M N .
$$

4. Update the weight vectors of the winner neuron $j^{*}$ and its neighboring neurons according to the following equations: 


$$
\begin{aligned}
& W_{i}(n+1)=W_{i}(n)+\eta(n)\left[X(n)-W_{i}(n)\right], \quad i \in S_{j^{*}}(n) \\
& W_{i}(n+1)=W_{i}(n), \quad i \notin S_{j^{*}}(n)
\end{aligned}
$$

where $\eta(n)$ is the learning rate such that $0<\eta(n)<1$, and $S_{j *}(n)$ is the neighborhood of the winner neuron $j^{*}$.

5. Repeat steps $2-4$ for $n=1, \ldots, N_{T}-1$, where $N_{T}$ is the total number of training vectors. This number should be at least 500 times more than the number of neurons $M N$ in the Kohonen network [8].

In order to improve the performance of the Kohonen network for our purpose, we suggest the following issues regarding the implementation of the learning process

1. For selecting appropriate values of $M$ and $N$, we can start with a large number, say $M=N=8$. In the next step, we change $M$ and $N$ such that $M N$ is approximately equal to the number of the neurons which have been activated.

2. The norm of the initial weight vectors have been chosen so as to fall within the range of the norms of training vectors.

3. The initial $S_{j *(0)}$ can be large enough to include all the $M N$ neurons in the network. If after several hundred training steps, neurons show an ordered pattern, $S_{j *}(n)$ can eventually shrink to contain only the winner neuron $j^{*}$. Let $M>N$. Several experiments have shown that the following $S_{j *}(n)$ is the best:

$$
\left|S_{j^{*}}(n)\right|=\left\{\begin{array}{ll}
g(n), & \text { if } g(n)>1 \\
1, & \text { if } g(n) \leq 1
\end{array},\right.
$$

where $\left|S_{*}(n)\right|$ is the size of the neighborhood and $g(n)=M(1 / M)^{10 n / N_{T}}$.

4. The learning rate $\eta(n)$, can be either a constant or a monotonically decreasing function. We suggest the following learning rate:

$$
\eta(n)=\frac{1}{1+L}\left(\frac{5}{4+n}+L^{n}\right) \quad L=\left(\frac{1}{N_{T}}\right)^{\frac{1}{\sqrt{1000 N_{T}}}} .
$$

5. Since we have used random initial weight vectors, after repeating the training procedure with the same training set $\left\{X(0), X(1), \ldots, X\left(N_{T}\right)\right\}$, similar weight vectors will be assigned to different neurons. For repeatability of the training results with the same training vector, the final weight vectors after the training procedure should be sorted.

At this time, the raw features of the input signal, namely the time-frequency distribution data, have been encoded into the weight vectors of the Kohonen network. Each weight vector represents a particular kind of input pattern. Thus different locations of the neurons in the Kohonen map reflect different input spaces, which provide us with the information required for clustering the test data. During the clustering of the test data, each neuron or a group of neighboring neurons in the Kohonen map responds to a particular kind of input pattern. So, we expect different types of transient input signals to activate neurons (winning neurons) in different locations of the Kohonen map. Consequently, we can use the index sequence of active neurons to discriminate between crack-related AE's and transient interference.

\section{CLUSTERING RESULTS OF DATA}

\subsection{In-flight data}

For the first set of data, a $5 \times 5$ Kohonen network was considered. The training set was composed of 2000 fivedimensional principal component vectors of the STFT's of the first half of the signal recorded by sensor 2 . In this case the window size and FFT point of the STFT are 1024. The test set consists of the second half of the signal of sensor 2, and all the signals of other sensors. Note that both the training and the test sets contain simulated cracks together with other interferences. Fig. 5 shows a small portion of the input test signal in timefrequency space, and Fig. 6 is the index of the corresponding activated neurons at the output of the Kohonen network. As can be inferred from these two figures, crack events (spikes in the time-frequency domain) activate neurons with high indices. This kind of behavior has been observed for other test signals, and shows the mechanism by which the Kohonen network successfully identifies cracks from strong interferences.

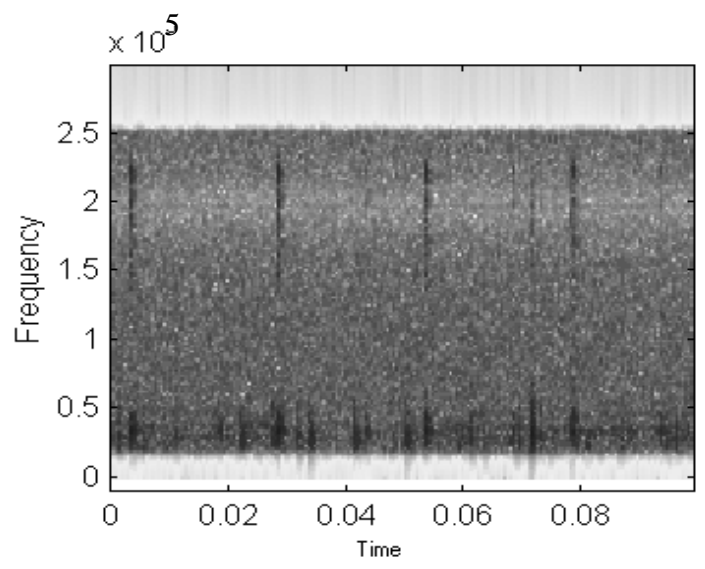

Figure 5. STFT of a segment of data from sensor 2.

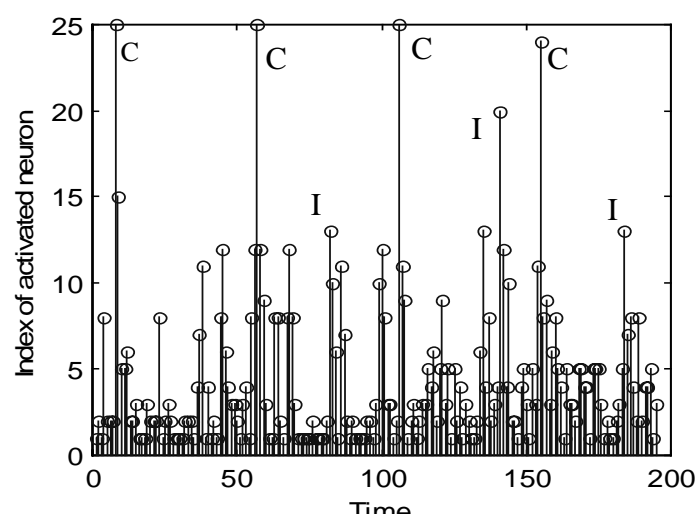

Figure 6. Indices of the activated neurons corresponding to the time-frequency components of the above segment. 
For sensor 2, the probability of incorrect identification was less than $4 \%$, i.e. more than $96 \%$ of simulated cracks were identified correctly. For other sensors (except sensor 3 which has corrupted, useless data), the probability of correct identification was in the range of $75 \%-85 \%$.

\subsection{Multi-sensor laboratory data}

For the second set of data, a $5 \times 5$ Kohonen network was again found to be suitable. The training set was composed of 200 fivedimensional principal component vectors of the STFT's, randomly chosen from the signal of sensor 1 . Because of the limited number of the available training vectors, we used them repeatedly during the training phase (so $N_{T}$ is the number of the training vectors, 200, plus the number of repeated vectors). The test set consists of the signals of all four sensors, with the training set excluded. Similar to the previous data set, both the training and the test sets contain real cracks together with other noises.

Since all four sensors are at approximately the same distance from the central notch, we expect the same time delay for the crack-related signals, which are generated at the central notch. Since the information of the time delays can help us in identifying cracks, we have estimated the time delays using a lowpass filter, followed by a threshold block. Based on this time delay estimation, the events that do not occur simultaneously in four sensors can be defined as noise. In addition, as we explained before, we know that as time goes on, the probability of crack generation increases. Based on these two criteria, we can evaluate the performance of the designed Kohonen network. Fig. 7 shows the result of Kohonen-network clustering for a test set, composed of 941 events at sensor 3.

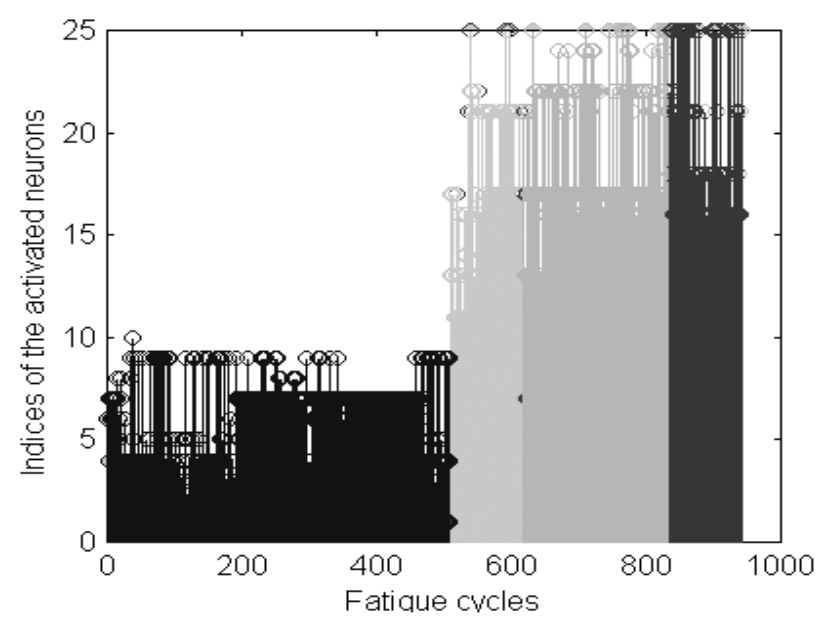

Figure 7. Indices of the activated neurons corresponding to the $\mathrm{AE}$ transients of sensor 3 .

This figure shows that, neurons with higher indices are activated as time increases. As a rule of thumb, we assume that an activated neuron whose index is higher than 20 corresponds to a crack-related signal. So, Fig. 7 is in agreement with the increase in the number of cracks as time progresses. The point of interest is that all neurons with indices higher than 20 correspond to simultaneous events in four sensors, as we expect.

\section{CONCLUSION}

In this paper we have proposed the application of the Kohonen neural network to the clustering of the acoustic emission signals for fault monitoring. Two different sets of data were used. Short- time Fourier transform of the data was employed as a useful feature for clustering. Principal component analysis was carried out to reduce the dimensionality of the data. Several suggestions were also made to improve the learning procedure, which in turn yields a more reliable network. Our results show that the Kohonen network is able to identify the crack-related signals in a high-noise/interference environment, with a small probability of error.

\section{ACKNOWLEDGEMENT}

We are grateful to Honeywell and the Mechanical Engineering Department of Georgia Institute of technology for providing us with the data. This work was supported by the Office of Naval Research under MURI contract \# N00014-95-1-0539

\section{REFERENCES}

[1] G. T. Venkatesan, D. Zhang, M. Kaveh, A. H. Tewfik, K. M. Buckly, "Signal Processing for Fault Monitoring using Acoustic Emission" AEU int.J.Elec.Comm. (accepted) 1999

[2] T. F. Drouillard, "A history of acoustic emission", J. Acoustic Emission, vol. 14, no. 1, 1996, pages 1-34.

[3] V. Cherkassky. F. Mulier, Learning from Data, 1998, Wiley \& Sons, INC.

[4] G. Venkatesan, D. West, K. Buckley, A Tewfik, M. Kaveh, "Automatic fault monitoring using acoustic emissions", ICASSP, Munich, April 1997, pages 573-576.

[5] H. Sun, M Kaveh, A. H. Tewfik, "Self-Organizing Map Neural Network for Transient Signal Classification in Mechanical Diagnostics" IEEE/EURASIP Workshop on Nonlinear Signal Processing, June 1999, Antalia, Turkey.

[6] K. Buckley, G. Venkatesan, D. West, M. Kaveh, "Detection and Characterization of cracks for failure monitoring and diagnostics", ICASSP, Atlanta, May 1996.

[7] L. Cohen, "Time-frequency distributions - a review", Proced. of IEEE, vol. 77, no. 7, July 1989, pages 941-981.

[8] T. Kohonen, "The self-organizing map", Proceeding of IEEE, vol.78, no. 9, Sept. 1990, pages 1464-1480.

[9] F. Malver, J. Schoess, J. Kooyman, R. Jiracek and J. Volk, "CH-46 Sea Knight flight-test results for the Rotor Acoustic Monitoring System (RAMS)", Honeywell Technology Center,

[10] L. Owsley, L. Atlas, and G. Bernard, "Feature extraction networks for dull tool monitoring", Proceeding of ICASSP 1995, pages 3355-3358.

[11] S. Haykin, Neural Networks, 1999, Prentice Hall

[12] D. Zhang, G. T. Venkatesan, , M. Kaveh, A. H. Tewfik, "Acoustic Emission Transient Detection Based on Linear Model Residual" 1998, Proc. IEEE SSAP Workshop, Portland,OR,Sept.,1998

[13] T. W. Anderson, An Introduction to Multivariate Statistical Analysis, 1984, J. Wiley \& Sons INC. 